\title{
DESCRIPCIÓN FONÉTICA DE LOS TONOS DEL MAYA YUCATECO
}

\author{
Martín Sobrino Gómez \\ Centro de Estudios Linguísticos y Literarios \\ El Colegio de México
}

Resumen: El presente escrito ofrece un análisis instrumental de las vocales del idioma maya yucateco y una descripción fonética de las realizaciones que manifiestan los tonos. En primer lugar se discriminan las vocales cortas de las largas y se pone especial énfasis en el análisis de las vocales largas con tonos fonológicos. Finalmente, se esbozan algunas consideraciones sobre los correlatos fonéticos del contraste tonal.

Palabras clave: realización fonética, tonos, maya yucateco.

Aвstract: The porpouse of the paper is to analysis of the vowels of Yucatec Maya, as well as a phonetic description of the realizations of tones. First, short vowels are distinguished from long vowels, and then special attention is paid to long vowels which show phonological tones. Finally, some considerations about the tonal contrast are outlined.

KEYWORDS: phonetic realizations, tones, yucatec maya.

ReCEPCIÓN: 12 de septiembre de 2011.

ACEPTACIÓN: 13 de febrero del 2012. 



\title{
DESCRIPCIÓN FONÉTICA DE LOS TONOS DEL MAYA YUCATECO
}

\author{
Martín Sobrino Gómez \\ Centro de Estudios Lingüísticos y Literarios \\ El Colegio de México
}

El maya yucateco es una lengua tonal hablada en la península de Yucatán, en México. Actualmente este idioma es hablado por 800291 personas en los tres estados mexicanos de Yucatán, Campeche y Quintana Roo (INEGI, 2001). También se encuentran hablantes de este idioma en los distritos de Corozal y Orange Walk en Belice. El maya yucateco está clasificado junto con el itzá (Guatemala), mopán (Belice y Guatemala) y lacandón (Chiapas) dentro de la rama yucatecana en la familia linguiística mayance (Kaufman, 1990).

El sistema consonántico del maya yucateco cuenta con resonantes y obstruyentes. Entre las resonantes encontramos nasales, laterales y aproximantes; y entre las obstruyentes, fricativas, oclusivas y africadas. Tanto en la serie de oclusivas como en las africadas existe un contraste entre sordas y sordas glotalizadas (tabla 1). ${ }^{1}$

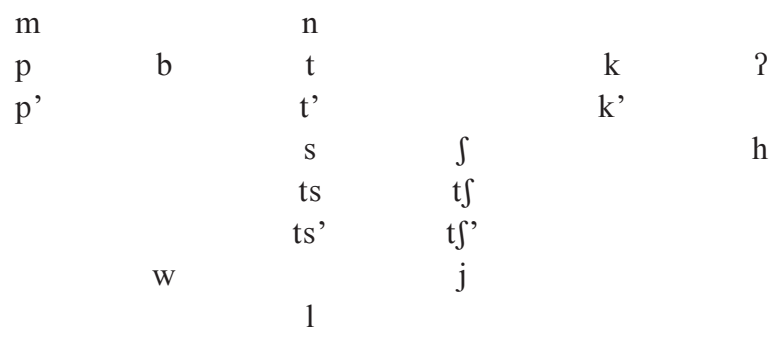

TABLA 1. Inventario de fonemas consonánticos del maya yucateco

\footnotetext{
${ }^{1}$ Existe una discusión a propósito del estatuto presentado como /b/ en la tabla 1 puesto que, de acuerdo con investigaciones recientes (Bennett, 2002; Frazier, 2009), este idioma tiene una consonante bilabial implosiva /6/ y no una oclusiva bilabial sonora /b/ o eyectiva /b\%. Sobre esto, Bennett (2002: 15) apunta que: "The voiced bilabial's manner of articulation is often undefined. In modern Yucatec Maya it is written as b' or b, depending on the orthography, leaving the possibility for an implosive, or a voiced stop followed by a glottal stop or some kind of glottalization". Para el dialecto de Sisbicchen, Yucatán, Frazier (comunicación personal) ha encontrado algunos ejemplos en los que esta consonante se encuentra en posición de coda silábica y se aprecia que "there is a stop release, but it does not look like an implosive" por lo que aún "there are lots of concerns... for future research". De esta manera, la presente investigación usará la grafía b (oclusiva bilabial sonora) del AFı para representar esta consonante sin entrar en más detalle sobre su articulación en las transcripciones fonéticas que se presenten a lo largo de este escrito.
} 
Este idioma posee cinco timbres vocálicos que son contrastivos (tabla 2). Existen otros rasgos en las vocales que también son contrastivos entre sí, como son la duración, el estado glótico y la tonalidad. En la mayoría de las investigaciones recientes sobre esta lengua se hace la distinción entre vocales breves, vocales largas y vocales rearticuladas. Muchos de los autores muestran acuerdo en que las vocales largas presentan tonos fonológicos.

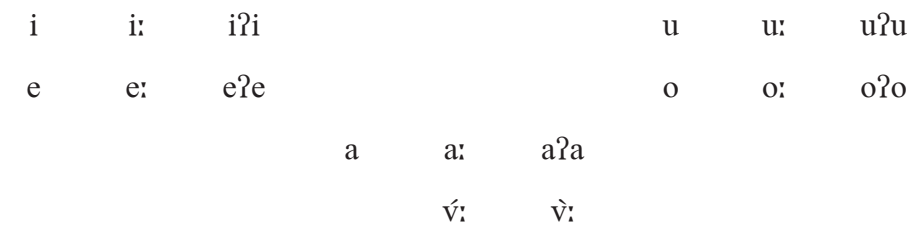

TABLA 2. Sistema vocálico y tonal del maya yucateco.

Para describir las vocales, y en especial los tonos del maya yucateco, se analizaron datos obtenidos de cuatro hablantes: dos hombres y dos mujeres. Una pareja es del municipio de Hopelchén, Campeche, y la otra del municipio de Hunucmá, Yucatán. ${ }^{2}$ Los cuatro colaboradores habitan en las cabeceras municipales de sus respectivos municipios. Son hablantes bilinguies maya-español y utilizan ambas lenguas en su vida cotidiana. El cuadro 1 muestra de manera resumida la información sobre los colaboradores. Ambos municipios se encuentran en el Noroeste de la península de Yucatán.

\begin{tabular}{|l|c|c|c|c|}
\hline \multirow{2}{*}{$\begin{array}{l}\text { Hopelchén, } \\
\text { Campeche }\end{array}$} & Colaborador 1 & Mujer & 64 años & inf1hopcam \\
\cline { 2 - 5 } & Colaborador 2 & Hombre & 67 años & inf2hopcam \\
\hline $\begin{array}{l}\text { Hunucmá, } \\
\text { Yucatán }\end{array}$ & Colaborador 1 & Mujer & 40 años & inf1hunyuc \\
\cline { 2 - 5 } & Colaborador 2 & Hombre & 58 años & inf2hunyuc \\
\hline
\end{tabular}

Cuadro 1. Los hablantes.

Los datos se obtuvieron a partir de la aplicación de cuestionarios que contenían listas de palabras que los informantes debían traducir a su lengua materna. Estos datos fueron almacenados en una grabadora digital de voz. Todas las grabaciones se editaron con ayuda del programa Adobe Audition 2.0 y los archivos de audio se analizaron con el programa PRAAT (Boersma y Weenik, 2008) para poder obtener transcripciones más fiables con el Alfabeto Fonético Internacional (AFI).

A los cuatro colaboradores se les aplicó un cuestionario de 392 ítems léxicos con el fin de obtener la mayor cantidad de datos posible. La lista incluyó pa-

\footnotetext{
${ }^{2}$ Nota del editor. Para agilizar la lectura del artículo en lo sucesivo se omitirá en la referencia a Holpechén y Hunucmá el nombre de los estados a los que pertenecen, Campeche y Yucatán, respectivamente.
} 
labras con el patrón silábico cvc cuyo núcleo silábico pudiera llevar cualquiera de los cuatro tipos de vocal: breve, larga con tono bajo, larga con tono alto y rearticulada. Todos los ítems léxicos fueron pronunciados por los informantes de dos a cuatro veces y la emisión elegida para su análisis fue la que se consideró pronunciada con mayor claridad.

\section{La longitud de las vocales}

El criterio para medir la duración de las vocales fue tomando en cuenta la estabilidad de la estructura formántica en el espectro. Se dio preferencia al análisis de palabras que tuvieran obstruyentes tanto en inicio de sílaba como en coda.

En los cuadros 2 y 3 podemos ver el promedio de la longitud de las vocales breves. El cuadro 2 presenta el promedio de cada hablante y el cuadro 3 muestra el promedio general.

\begin{tabular}{|l|c|}
\hline & cve \\
\hline inf1hopcam & 119 \\
\hline inf2hopcam & 113 \\
\hline inf1hunyuc & 106 \\
\hline inf2hunyuc & 97 \\
\hline
\end{tabular}

CuAdro 2. Promedio de duración de la vocal breve por hablante (ms).

\begin{tabular}{|l|}
\hline cve \\
\hline 108 \\
\hline
\end{tabular}

CuAdro 3. Promedio general de duración de la vocal breve (ms).

Las vocales largas tienen un rango de duración mucho más amplio que el de las vocales breves. El análisis de nuestros datos deja ver que la duración parece depender del rasgo suprasegmental de la vocal, es decir, si tiene tono alto, tono bajo o si es rearticulada. El tiempo de realización de las vocales largas con tono bajo es menor que el de las vocales largas con tono alto; ${ }^{3}$ por su parte, el tiempo de duración de las vocales rearticuladas es mayor que el de las vocales largas con tono bajo, pero menor al de las vocales largas con tono alto. En el cuadro 4 se ilustran los promedios de duración de estas vocales que se obtuvieron por cada hablante.

\footnotetext{
${ }^{3}$ Uno de los dictaminadores ha hecho notar que en la literatura sobre la relación entre duración vocálica y tonos se indica que los tonos altos tienden a manifestarse en porciones vocálicas más breves que los tonos bajos, y que el hallazgo de la situación contraria en el maya yucateco constituye una aportación empírica de gran interés tanto descriptivo como teórico.
} 


\begin{tabular}{|l|c|c|c|}
\hline & cv̀:c & cv́:c & cv?vc \\
\hline inf1hopcam & 195 & 219 & 218 \\
\hline inf2hopcam & 235 & 274 & 227 \\
\hline inf1hunyuc & 205 & 257 & 251 \\
\hline inf2hunyuc & 152 & 213 & 200 \\
\hline
\end{tabular}

Cuadro 4. Promedio de duración de las vocales largas por hablante (ms).

En el cuadro 5 se muestran los promedios de las vocales largas y se dividen por el tipo de rasgo suprasegmental.

\begin{tabular}{|l|c|c|c|}
\hline Tipo de rasgo suprasegmental & cv̀:c & cv́:c & cv?vc \\
\hline Duración & 196 & 240 & 224 \\
\hline
\end{tabular}

Cuadro 5. Promedio de duración de las vocales largas (ms)

A continuación veremos algunos ejemplos donde se contrasta la diferencia en la duración de la vocal. En cada figura se expone en primer lugar la palabra que lleva vocal larga, y en segundo, la palabra con vocal breve. Los espectrogramas que se ilustran son del habla del colaborador hombre de Hopelchén. La línea de las ilustraciones representa la intensidad.

La figura 1 muestra la distinción léxica de la longitud entre las palabras chiich 'abuela' y chich 'duro'. La duración de la vocal en chiich 'abuela' es de 255 ms, mientras que la duración de la palabra chich 'duro' es de 105 ms.
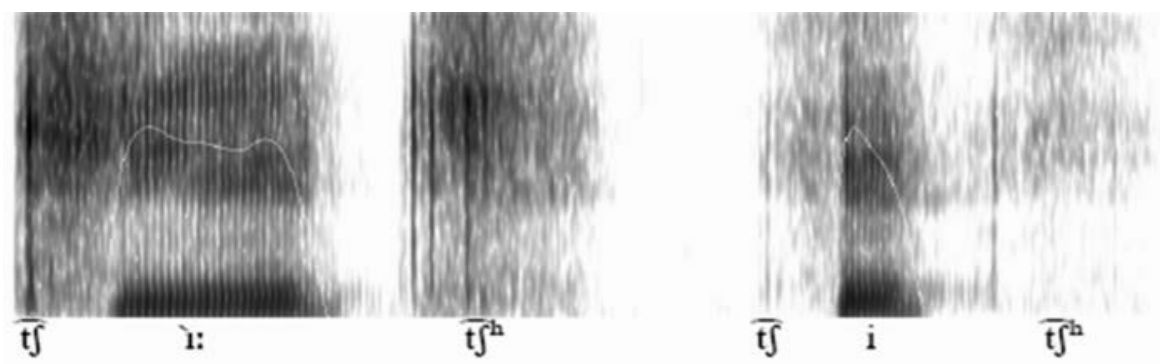

FiguRA 1. Distinción en la duración vocálica chiich-chich ('abuela'-'duro').

La figura 2 presenta la distinción léxica de la longitud entre báat 'hacha' y bat 'granizo'. La vocal de la palabra báat 'hacha' tiene una duración de $303 \mathrm{~ms}$, en tanto que la de la palabra bat 'granizo' es de125 ms. 

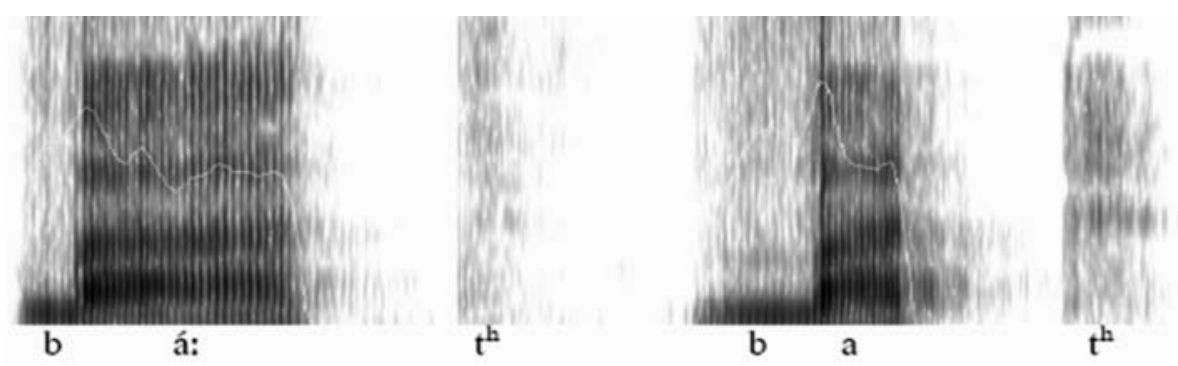

Figura 2. Distinción en la duración vocálica báat-bat ('hacha'-'granizo').

\section{Realización fonética de los tonos}

Los tonos se midieron tomando como inicio de los mismos la estabilidad tonal, es decir, no se consideró la influencia que pudiera causar una consonante a inicio de palabra. ${ }^{4}$

\section{Tono bajo}

Este tono presenta dos realizaciones fonéticas: una nivelada y otra ascendente. En el cuadro 6 se muestran los valores en Hertz del tono bajo en su realización nivelada por cada colaborador. Podemos proponer el promedio para el tono bajo en voz de hombre entre los $115 \mathrm{~Hz}$ y los $120 \mathrm{~Hz}$; y para la voz de mujer, entre los $180 \mathrm{~Hz}$ y los $190 \mathrm{~Hz}$.

\begin{tabular}{|l|c|}
\hline Informante & $\mathbf{H z}$ \\
\hline inf1hopcam & 190 \\
\hline inf2hopcam & 120 \\
\hline inf1hunyuc & 180 \\
\hline inf2hunyuc & 115 \\
\hline
\end{tabular}

Cuadro 6. Promedio en $\mathrm{Hz}$ del tono bajo nivelado por hablante.

La realización ascendente del tono bajo inicia su trayectoria con aproximadamente los mismos valores que se tienen en la realización nivelada y la finaliza con aproximadamente los mismos valores que se obtuvieron para el tono alto en

\footnotetext{
${ }^{4}$ En la informante mujer de Hopelchén se analizaron 59 ítems léxicos para la vocal larga con tono bajo y 74 para la vocal larga con tono alto. En el informante hombre de Hopelchén se analizaron 44 ítems léxicos para la vocal larga con tono bajo y 54 para la vocal larga con tono alto. En la informante mujer de Hunucmá se analizaron 38 ítems léxicos para la vocal larga con tono bajo y 50 para la vocal larga con tono alto. En el informante hombre de Hunucmá se analizaron 33 ítems léxicos para la vocal larga con tono bajo y 36 para la vocal larga con tono alto.
} 
su realización nivelada (presentados en el siguiente apartado). El rango en Hertz del punto más bajo al más alto en la trayectoria tonal fue de $31 \mathrm{~Hz}$ a $42 \mathrm{~Hz}$, excepto por el colaborador hombre de Hunucmá, en cuya única emisión donde el tono se realizó ascendente el rango fue de $22 \mathrm{~Hz}$.

En el cuadro 7 se presentan por cada hablante los promedios inicial y final en Hertz de la realización del tono bajo como contorno ascendente.

\begin{tabular}{|l|c|c|c|}
\hline Colaborador & Inicio & Final & Diferencia \\
\hline inf1hopcam & 182 & 224 & 42 \\
\hline inf2hopcam & 114 & 145 & 31 \\
\hline inf1hunyuc & 184 & 220 & 36 \\
\hline inf2hunyuc & 125 & 147 & 22 \\
\hline
\end{tabular}

CuAdro 7. Promedios inicial y final en $\mathrm{Hz}$ del tono bajo en su realización ascendente.

Tono alto

El tono alto manifestó tres realizaciones fonéticas: una nivelada, una descendente y otra compleja. En el cuadro 8 se muestran los promedios en Hertz que tiene este tono realizado de manera nivelada por cada hablante. Los valores que se pueden proponer para este tono realizado como un tono de nivel alto son de 122 $\mathrm{Hz}$ a $145 \mathrm{~Hz}$ para la voz de hombre y de $210 \mathrm{~Hz}$ a $215 \mathrm{~Hz}$ para la voz de mujer.

\begin{tabular}{|l|c|}
\hline Informante & Hz \\
\hline inf1hopcam & 215 \\
\hline inf2hopcam & 145 \\
\hline inf1hunyuc & 210 \\
\hline inf2hunyuc & 122 \\
\hline
\end{tabular}

Cuadro 8. Promedio en $\mathrm{Hz}$ del tono alto nivelado por hablante.

La realización descendente del tono alto inicia su trayectoria con aproximadamente los mismos valores que se tienen en la realización nivelada del mismo $\mathrm{y}$, al igual que en el caso de la realización ascendente del tono bajo, finaliza con aproximadamente los mismos valores que se obtuvieron para la realización nivelada del tono bajo. El rango en Hertz del punto más bajo al más alto en la trayectoria tonal fue de $24 \mathrm{~Hz}$ a $53 \mathrm{~Hz}$.

En el cuadro 9 se presentan por cada hablante los promedios inicial y final en Hertz de la realización del tono alto como contorno descendente. 


\begin{tabular}{|l|c|c|c|}
\hline Colaborador & Inicio & Final & Diferencia \\
\hline inf1hopcam & 220 & 167 & 53 \\
\hline inf2hopcam & 138 & 107 & 31 \\
\hline inf1hunyuc & 208 & 163 & 45 \\
\hline inf2hunyuc & 129 & 105 & 24 \\
\hline
\end{tabular}

Cuadro 9. Promedios inicial y final en $\mathrm{Hz}$ del tono alto en su realización descendente.

La forma compleja del tono alto se ubicó en dos de los colaboradores: en el hombre de Hopelchén y en la mujer de Hunucmá. En el hombre de Hopelchén la realización es descendente-ascendente. El promedio de los valores que se obtuvieron de estas realizaciones está entre los 130-100-150 Hz, aunque algunos valores también aparecieron entre los 160-140-180 Hz; sin embargo, es notable que cuando el tono vuelve a ascender la frecuencia final es mayor que la del punto inicial de la trayectoria tonal. En la mujer de Hunucmá esta realización del tono también es descendente-ascendente; el promedio de los valores de esta realización es de 220-170-200 Hz, y puede notarse que, a diferencia del hablante de Campeche, cuando el tono asciende nuevamente, la frecuencia no supera la del punto inicial de la trayectoria.

En el cuadro 10 se ilustran los porcentajes de la frecuencia con la que aparecen en cada hablante las realizaciones del tono bajo y del tono alto en todos los ítems estudiados. De esta manera se observa que el tono alto se manifestó mayormente con forma descendente en la hablante mujer de Hopelchén, Campeche, y en los dos hablantes de Hunucmá, Yucatán, en tanto que para el hablante hombre de Hopelchén, Campeche, la realización más frecuente de este tono fue descendenteascendente. Se observa igualmente que esta última realización aparece, aunque en menor medida, en la hablante de Hunucmá, Yucatán. Por su parte, la forma nivelada del tono bajo predomina en los dos hablantes de Hunucmá, Yucatán, y en el hablante hombre de Hopelchén, Campeche, mientras que la forma ascendente de este tono fue preferente en la hablante mujer de Hopelchén, Campeche.

\section{Contraste léxico tonal}

En este apartado se expondrá una de las funciones de los tonos en el maya yucateco. Veremos cómo el uso de uno u otro tono distingue el significado de algunas palabras a nivel léxico. Se presentan los espectrogramas de palabras monosilábicas cuyo contraste se debe al tono que lleva la vocal. Los ejemplos se obtuvieron de la informante mujer de Hopelchén y del informante hombre de Hunucmá.

Hemos observado que el contraste que presenta la informante de Hopelchén es entre un contorno ascendente y un contorno descendente, y de hecho notamos en las figuras 3 y 4 que la realización ascendente tiene la frecuencia más alta que la 


\begin{tabular}{|c|c|c|c|c|}
\hline $\begin{array}{l}\text { Realización } \\
\text { fonética }\end{array}$ & nivelado & ascendente & descendente & $\begin{array}{l}\text { descendente- } \\
\text { ascendente }\end{array}$ \\
\hline $\begin{array}{l}\text { inf1hopcam } \\
\text { tono bajo } \\
\text { tono alto }\end{array}$ & $\begin{array}{l}44 \% \\
16 \%\end{array}$ & $56 \%$ & $84 \%$ & \\
\hline $\begin{array}{l}\text { inf2hopcam } \\
\text { tono bajo } \\
\text { tono alto }\end{array}$ & $\begin{array}{l}66 \% \\
13 \% \\
\end{array}$ & $\begin{array}{c}34 \% \\
7 \%\end{array}$ & $28 \%$ & $52 \%$ \\
\hline $\begin{array}{l}\text { inf1hunyuc } \\
\text { tono bajo } \\
\text { tono alto }\end{array}$ & $\begin{array}{c}81.5 \% \\
26 \% \\
\end{array}$ & $18.5 \%$ & $64 \%$ & $10 \%$ \\
\hline $\begin{array}{l}\text { inf2hunyuc } \\
\text { tono bajo } \\
\text { tono alto }\end{array}$ & $\begin{array}{l}97 \% \\
33 \%\end{array}$ & $3 \%$ & $67 \%$ & \\
\hline
\end{tabular}

CuAdro 10. Porcentajes de la realización fonética de los tonos por hablante.

realización descendente, lo que sugiere la importancia que tienen las realizaciones de los tonos en la lengua. Con el informante de Hunucmá vemos que sus contrastes son de un tono de nivel contra un contorno descendente. Es importante señalar que, además de la diferencia en las realizaciones de los tonos para el contraste, la diferencia en la longitud vocálica en ambos hablantes también es evidente.

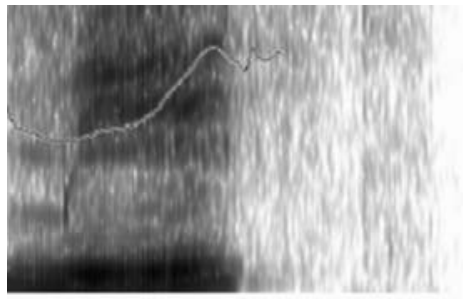

$\mathbf{m}$ ц: s

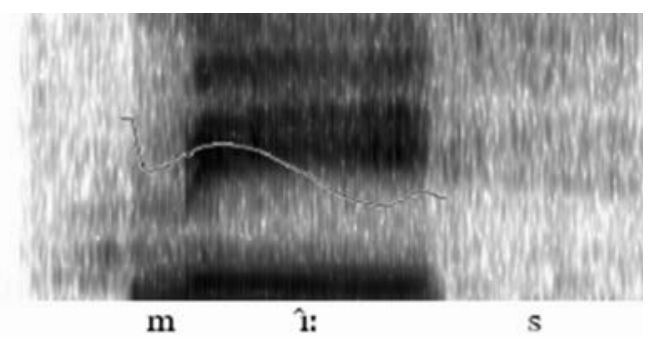

FIGURA 3. Contraste tonal entre las palabras 'gato' y ‘escoba' (mujer).
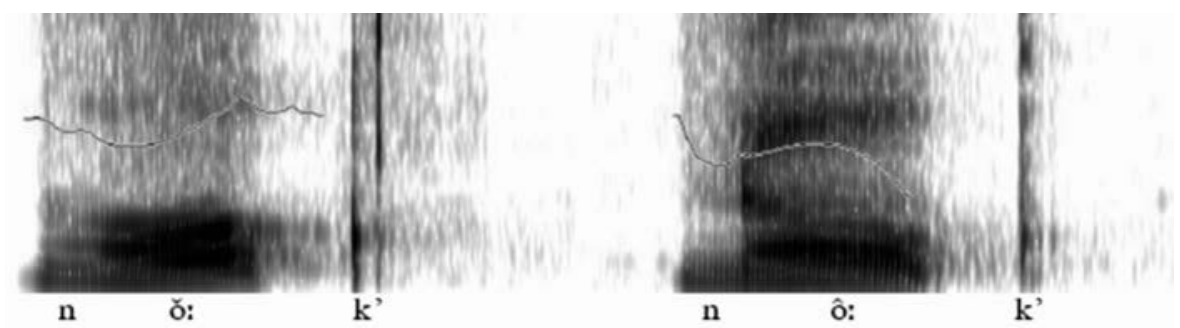

FIGURA 4. Contraste tonal entre las palabras 'ropa' y 'roncar' (mujer). 


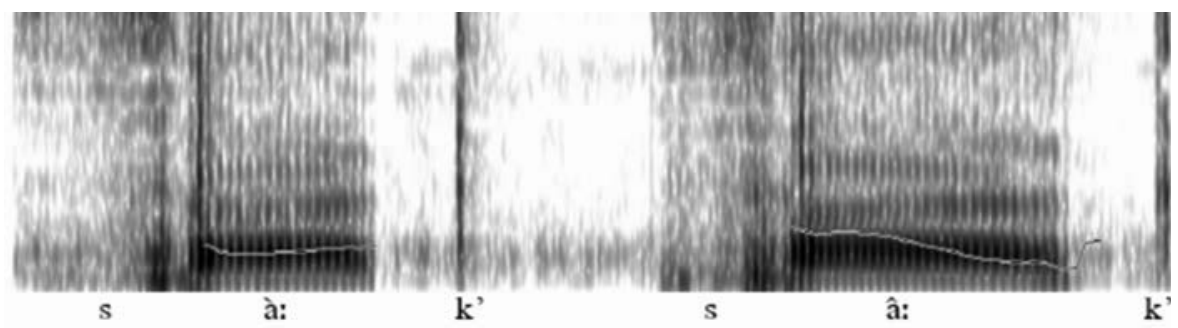

Figura 5. Contraste tonal entre las palabras ‘comezón’ y ‘langosta’ (hombre).

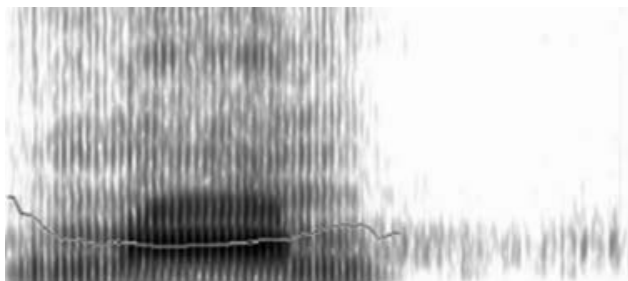

$\mathrm{m}$ à: $\mathbf{n}$

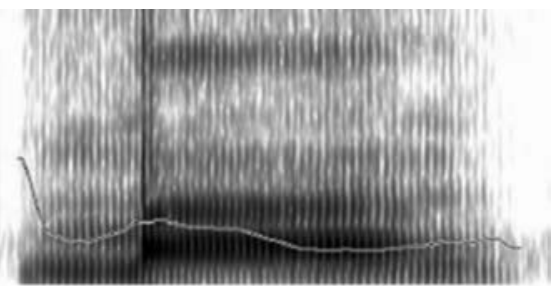

$\mathrm{m}$ â: $\mathbf{n}$

Figura 6. Contraste tonal entre las palabras 'comprar' y 'pasar' (hombre).

\section{Realizaciones tonales en emisiones bisilábicas y trisilábicas}

Para complementar la descripción fonética de los tonos en el maya yucateco se tomaron en cuenta emisiones bisilábicas y trisilábicas en las que los tonos léxicos se encontraban en diferentes posiciones de la emisión. En dichos patrones silábicos, el tono bajo se realizó constantemente como un tono de nivel; por su parte, el tono alto muestra más bien una realización descendente. Algunos ejemplos tomados de la informante mujer de Hunucmá se presentan enseguida.

En el cuadro 11 se presentan, de manera resumida, los patrones silábicos en los que pueden manifestarse las distintas realizaciones de los tonos de este idioma de acuerdo con nuestros datos.

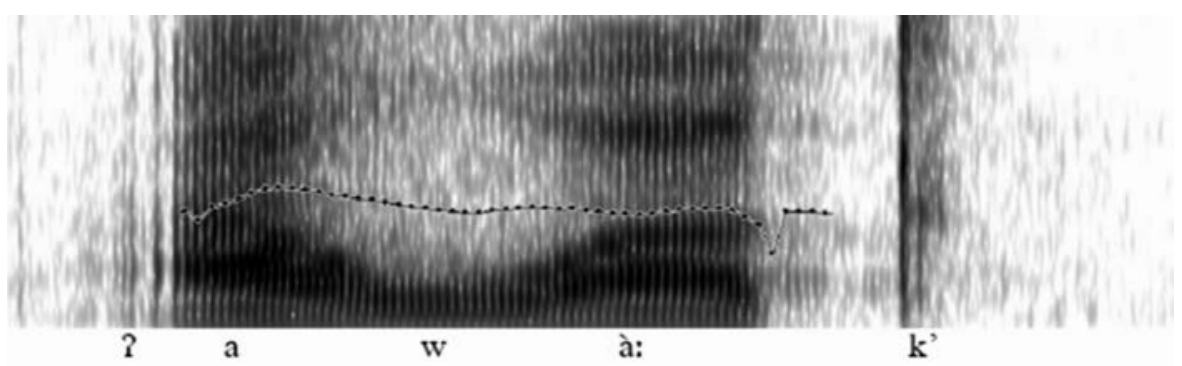

FIGURA 7. Realización del tono bajo en la frase $a w=a a k$ ' tu lengua'. 


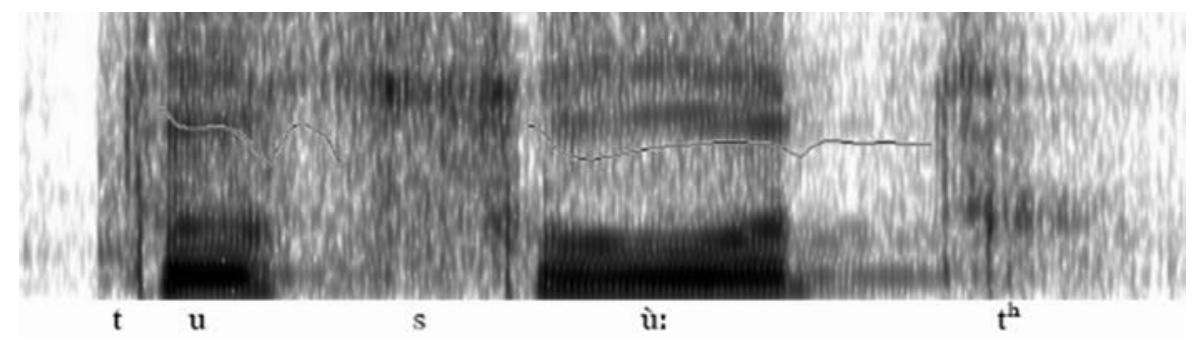

Figura 8. Realización del tono bajo en la frase $t$ - $u=$ suut 'está regresando'.

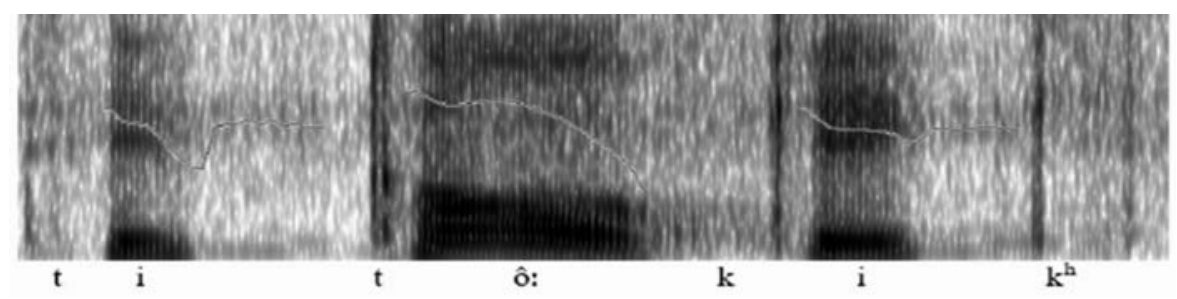

FIGURA 9. Realización del tono alto en la frase $t$-in=tóok-ik 'lo estoy quemando'. 


\begin{tabular}{|c|c|c|c|c|c|}
\hline $\begin{array}{c}\text { Tono } \\
\text { fonológico }\end{array}$ & Tor & o bajo & & Tono alto & \\
\hline $\begin{array}{l}\text { Realización } \\
\text { fonética }\end{array}$ & Nivelada & Ascendente & Nivelada & Descendente & $\begin{array}{l}\text { Descendente- } \\
\text { ascendente }\end{array}$ \\
\hline \multirow{3}{*}{$\begin{array}{l}\text { Patrón } \\
\text { silábico }\end{array}$} & cù:c & \multirow[t]{3}{*}{ cV̌:c } & \multirow[t]{3}{*}{ cv́:c } & ĉे:c & cṽ:c \\
\hline & cv̀:c(c)ve & & & cĥ:c(c)ve & \multirow[t]{2}{*}{ cveṽ:c } \\
\hline & cv(c)cv̀:c & & & cvê̂:c & \\
\hline
\end{tabular}

CuAdro 11. Realización fonética de los tonos fonológicos del maya yucateco.

Este comportamiento parece repetirse en estudios en los que se toma en cuenta el lugar que la palabra con tono ocupa en la oración con el fin de conocer si los diversos contextos influyen de alguna manera. Por ejemplo, Kügler y Skopeteas (2006) investigan si las construcciones de focalización y topicalización están asociadas con eventos tonales particulares. Con este fin ellos analizan la realización de los tonos en palabras (monosilábicas y bisilábicas) en distintas posiciones dentro de la oración: a) en oraciones donde la palabra en cuestión se encuentra como un argumento simple del verbo existencial, b) en oraciones donde la palabra en cuestión se encuentra en posición de foco y c) en oraciones donde la palabra en cuestión se encuentra en posición de tópico.

Sus resultados muestran que no existe evidencia para asociar eventos tonales con la estructura de la información de la oración. Con respecto a los rasgos de los tonos, Kügler y Skopeteas (2006) describen que el tono bajo se realiza como un tono bajo de nivel (low level pitch). En palabras bisilábicas con tono bajo en la primera sílaba y tono alto en la segunda, el primero se realiza como un tono bajo de nivel y el segundo de manera ascendente (rising pitch). En resumen, ellos argumentan que el tono bajo siempre se presenta nivelado en tanto que el tono alto puede realizarse como un contorno ascendente (rising) o como un tono alto de nivel (high level pitch), pero nunca descendente (falling).

Sin embargo, Kügler y Skopeteas (2006) asumen que un tono alto puede caer si la unidad portadora de tono ocurre a final de frase. Debido a esto ellos explican que una palabra pronunciada de manera aislada tiene su propia entonación de frase, y es de esta forma que analizan algunos ejemplos de palabras bisilábicas donde ambas sílabas llevan tono alto y atribuyen la caída tonal de la segunda sílaba a la "interaction between a lexical high tone and a low intonation phrase boundary, thus an interaction between tone and intonation" (Kügler y Skopeteas 2006: 386). 
De manera similar, Gussenhoven y Teew (2008) analizan los "hechos fonéticos y fonológicos" de los tonos del yucateco tomando en cuenta los efectos de la posición de las palabras en la oración. De acuerdo con sus resultados, las palabras con tono alto manifiestan una caída a final de oración y un ascenso a inicio. Las palabras con tono bajo muestran ser estables tanto a inicio como a final de la oración.

Frazier (2009) describe para las comunidades de Mérida y Santa Elena las realizaciones de los tonos bajo y alto para el maya yucateco. Explica que el tono bajo se mantiene firme mientras que el tono alto comienza con una tonía alta y termina con una tonía baja. Menciona que para el maya de Sisbicchen ambos tonos presentan el mismo contorno ascendente. Para la variante de esta última comunidad esta autora propone que no existe un contraste léxico basado en el tono.

\section{Consideraciones sobre los correlatos fonéticos del contraste tonal en maya yucateco}

En la mayoría de las investigaciones recientes sobre el maya yucateco, los autores han propuesto un sistema tonal binario que consta de un tono alto y de un tono bajo (Pike, 1946; Blair, 1966; McQuown, 1970; Fisher, 1976; Bricker, 1981; Kaufmann, 1990; Küegler y Skopeteas, 2006; Gussenhoven y Teew, 2008, Frazier, 2009), a excepción de Fox (1978), quien habla de un tono bajo de nivel versus un tono descendente, y de Bennett (2002), quien propone que el sistema se compone de un tono de contorno y de uno de nivel.

La tipología señala que en lenguas con un sistema de dos tonos generalmente se contrastan dos tonos de nivel más que uno ascendente y uno descendente (Yip 2002: 25). ${ }^{5}$ Si bien en la superficie cada tono del maya yucateco puede tener más de una realización fonética, esta lengua parece ser del tipo en el que todos los hechos tonales se pueden analizar como "tonos puntuales" (Martinet, 1965: 110).

En lo fonológico, los hablantes de maya yucateco contrastan dos tonos de nivel: uno alto y otro bajo. No obstante, en la superficie pueden manifestarse un tono bajo o un tono ascendente correspondientes con el tono bajo del sistema, y un tono alto, un tono descendente o hasta un tono cóncavo correspondientes con el tono alto del sistema subyacente. No existe contraste fonológico entre la realización nivelada baja y la ascendente, o entre la realización nivelada alta y las formas descendente y compleja, ni entre éstas dos últimas. El contraste se da solamente entre las realizaciones correspondientes con el tono bajo y las correspondientes con el tono alto. Parte de esta alotonía se debe a que sólo las vocales largas son las que pueden llevar un tono fonológico. Para explicar este comportamiento se propone que la unidad portadora de tono en el maya yucateco es la mora $(\mu)$.

\footnotetext{
${ }^{5}$ Se han reportado algunas otras excepciones de lenguas con dos tonos que no contrastan tonos de nivel, como por ejemplo, Maddieson (1978) reporta que el Amahuaca contrasta un contorno ascendente y un contorno descendente; asimismo, Can Pixabaj (2007: 49) reporta para el Uspanteko la existencia de un tono alto y de un contorno descendente.
} 
De esta forma las vocales largas, que son bimoráicas, tienen dos unidades portadoras de tono y a cada una se le asocia el tono como se representa en (1). Para el caso en que el tono alto se realiza de forma nivelada, las dos moras se encuentran asociadas con un tono alto (A) como en (1a) o con un tono bajo (B) como en (1b); cuando el tono se realiza de forma descendente, la primera mora se asocia con un tono alto y la segunda con un tono bajo, como está representado en (1c), mientras que cuando el tono es ascendente, la primera mora se encuentra asociada con un tono bajo y la segunda con un tono alto como en (1d).

Cuando el tono es cóncavo la primera mora se asocia a dos tonos, uno alto y uno bajo, y la segunda mora con un tono alto como en (1e), o bien, la primera mora se encuentra asociada con un tono alto y la segunda mora con un tono bajo y uno tono alto como en (1f).

\begin{tabular}{|c|c|c|}
\hline \multicolumn{3}{|l|}{ (1) } \\
\hline a. cVvc & b. crvc & c. CVVc \\
\hline$\Lambda$ & 11 & $/ 1$ \\
\hline$\mu \mu$ & $\mu \mu$ & $\mu \mu$ \\
\hline V & V & 11 \\
\hline A & B & A B \\
\hline d. cvvc & e. crvc & f. cvvc \\
\hline 八 & $/ 1$ & 11 \\
\hline$\mu \mu$ & $\mu \mu$ & $\mu \mu$ \\
\hline 11 & $\wedge 1$ & $1 \wedge$ \\
\hline B A & $\mathrm{AB} A$ & A BA \\
\hline
\end{tabular}

Al parecer, el hecho de que esta lengua tenga un sistema tonal simple, de solamente dos tonos, ofrece mayor libertad para su realización. En nuestro análisis, las diversas realizaciones de los tonos no parecen relacionarse con algún contexto fonético determinado. Sin embargo, al tomar en cuenta los datos estadísticos de la frecuencia con que aparece cada realización en los colaboradores se encuentra mayor sistematicidad. Esto es, si tomamos en cuenta qué realización del tono pronunció cada hablante en la mayoría de sus emisiones veremos que cada uno de ellos tiene preferencia por alguna de las realizaciones tonales ya descritas (cuadro 10).

De esta manera, la hablante mujer de Hopelchén contrasta fonéticamente un tono ascendente $(\mathrm{BA})$ con un tono descendente $(\mathrm{AB})$, el hablante de Hopelchén contrasta un tono bajo (B) con un tono cóncavo (ABA), la hablante mujer de Hunucmá contrasta un tono bajo (B) con un tono descendente (AB), y el hablante hombre de Hunucmá un tono bajo (B) con un tono descendente (AB). La pareja de Hunucma comparte el mismo patrón tonal en tanto que la de Hopelchén presenta patrones distintos pero que no entorpecen la comunicación entre ellos y, probablemente, tampoco entre ambas parejas. 


\section{BIBLIOGRAFÍA}

Bennett, Lisa

2002 “Tone in Yucatec Maya”, tesis de maestría en Artes. Tulane: Tulane University.

Blair, Robert Wallace

1966 "Yucatec Maya Noun and Verb Morpho-Sintax". Tesis de doctorado. Indiana: Indiana University Microfilms, Department of Linguistics / Inc. Ann Arbor.

Boersma, Paul y David Weenink

2009 Praat: doing phonetics by computer (Version 5.1.04) [Computer program]. Retrieved April 4, 2009 <http://www.praat.org/>.

Bricker, Victoria R.

1981 Yucatec Maya Verbs (Hocaba Dialect) Gramatical Introduction, traducción de James Ward. Tulane: Tulane University. Aids Center for Latin America Studies. Latin American Studies Curriculum.

Can Pixabaj, Telma Angelina

2007 Jkemik yoloj li Uspanteko. Gramática Uspanteka. Guatemala: OKMA / ELDP. Cholsamaj Fundación.

Fisher, Willian M.

1976 "On tonal features in Yucatan dialects", Mayan Lingüistics (1): 29-43. Los Angeles: Marlys McClaran / American Indian Studies Center.

Fox, James Allan

1978 "Proto-Mayan Accent, Morpheme Structure Conditions, and Velar Innovations", tesis de doctorado. Chicago: University of Chicago.

Frazier, Melissa

2009 "Tonal Dialects and Consonant-Pitch Interactions in Yucatec Maya", New Perspectives in Mayan Linguistics, Heriberto Avelino, Jessica Coon y Elizabeth Norcliffe (eds.). Cambridge: Massachusetts Institute of Technology (Miт Working Papers in Linguistics, 59), 59-82.

Gussenhoven, Carlos y Renske Teeuw

2008 "A moraic and a syllabic H-tone in Yucatec Maya”, Fonología instrumental: patrones fónicos y variación lingüística, Esther Herrera y Pedro Martín Butragueño (eds.). México: Colegio de México, 49-72.

Instituto Nacional de Estadística, Geografía e Informática (INEGI)

2001 XII Censo Nacional de Población y Vivienda 2000. México: INEGI.

Kaufman, Terrence

1990 "Algunos rasgos estructurales de los idiomas mayances con referencia especial al k'iche”", Lecturas sobre la lingüística maya, Nora C. England y Stephen R. Elliot (comps.). Guatemala: Centro de Investigaciones Regionales de Mesoamérica, 59-114. 
Kügler, Frank y Stravros Skopeteas

2006 "Interaction of lexical tone and information structure in Yucatec Maya", Trends of Applied Linguistics. Berlín: De Gruyter, 380-388.

Maddieson, Ian

1978 "Universals of Tone”, Universal of Human Language, vol. 2 (Phonology), Joseph Greenberg (ed.) Standford CA: Standford University Press, 335-368.

Martinet, André

1965 Elementos de lingüística general. Madrid: Gredos.

McQuown, Norman

1970 "El Acento del Maya Yucateco". Ponencia presentada en el XXXIX Congreso Internacional de Americanistas: Lingüística e indigenismo moderno de América. Lima, Perú.

Pike, Kenneth L.

1946 "Phonemic pitch in Maya", International Journal of American Linguistics, 12 (2): 82-88. Chicago: University of Chicago.

Yip, Moira

2002 Tone. Cambridge: Cambridge University Press (Cambridge Textbooks in Linguistics). 
